\title{
Geographical epidemiology of residence of patients with motor neuron disease in Lancashire and south Cumbria
}

\author{
J D Mitchell, A C Gatrell, A Al-Hamad, R B Davies, G Batterby
}

Department of

Neurology, Royal Preston Hospital, Sharoe Green Lane,

Fulwood, Preston, UK

J D Mitchell

G Batterby

\section{Department of \\ Geography \\ A C Gatrell}

Centre for Applied Statistics, University of Lancaster, Lancaster,

UK

A Al-Hamad

R B Davies

Correspondence to: Professor J D Mitchell, Department of Neurology, Royal Preston Hospital, Sharoe Green Lane, Fulwood, Preston, PR2 9HT, UK. Telephone 00441772

710423, fax 00441772

718746 .

Received 10 Septenber 1997 and in final form

3 July 1998

Accepted 24 July 1998

\begin{abstract}
Objectives-To seek objective evidence for geographical clustering of places of residence of patients with motor neuron disease (MND).

Methods-A complete residential history from birth to onset of disease was obtained from a cohort of 130 patients with MND from Lancashire and south Cumbria presenting to the Department of Neurology in Preston between 1 January 1989 and 31 December 1993. These data were compared with population based reference data from the 1991 UK Census.

Results-Some areal units showed a greater, others a lesser, number of MND patient residences than expected. The results suggest that the background population incidence of MND is relatively low and that the overall incidence figures previously quoted have been skewed upwards by areas in which the incidence of MND is relatively increased. These findings were further tested by Poisson modelling. The Poisson model provided a poor fit for the data at postcode district and sector levels confirming that patients with MND were significantly more likely to have lived in some areas than others after allowing for variation in population of the different areal units and for variation in duration of residence.

Conclusions-These findings reinforce the results of previous work, much of which has been qualitative rather than quantitative. The results presented here suggest a low background incidence of MND in the context of generally quoted overall incidence figures. This low background incidence is, however, skewed upwards by some areal units with a relatively high incidence, thus achieving overall incidence rates comparable with generally quoted figures. We conclude that there is prima facie evidence of spatial patterns in the distribution of places of residence of patients with MND. Further examination of occupational and environmental factors in the lives of the patients with MND is required to obtain a better understanding of the importance of these findings.

(F Neurol Neurosurg Psychiatry 1998;65:842-847)
\end{abstract}

Keywords: amyotrophic lateral sclerosis; motor neuron disease; clusters
Health professionals, carers, and others involved in the care of patients with motor neuron disease (MND) repeatedly draw attention to a perception that the disease tends to occur within geographical clusters with respect to place of residence. These findings have often been anecdotal. Until recently it has been difficult to assess these impressions objectively. Further, whereas these anecdotal findings have tended to relate to relatively small areal units, previous work attempting to examine the geographical distribution of MND more objectively has been based on relatively large areal subunits. ${ }^{1}$ Although an earlier retrospective study of the geographical distribution of MND over a 10 year period in Lancashire and south Cumbria $^{2}$ suggested small area aggregation of cases, the methodology used in this work was based entirely on place of residence at the onset of disease.

After this earlier work we decided to embark on a prospective study to investigate place of residence of patients with MND throughout life, from birth to onset of disease in a specific population over a defined 5 year period.

\section{Methods}

We attempted to identify all patients presenting with MND in Lancashire and south Cumbria over the 5 year period 1 January 1989 to 31 December 1993. This period was chosen because it straddled the 1991 UK Census, which provided population based reference data. As this project was designed before the advent of the Escorial Criteria ${ }^{3}$ for the diagnosis of MND the diagnosis of the disease was established on the basis of detailed clinical assessment and full inpatient investigation including EMG, nerve conduction studies, muscle biopsy, CT , myelography, CSF examination, immunoelectrophoresis, glucose tolerance test, and thyroid function as considered appropriate by the consultant neurologist in charge to exclude syndromes mimicking MND. All patients were seen by at least two consultant neurologists during this evaluation before a definitive diagnosis of MND was made.

All patients were visited by a trained research sister (GB) and a detailed questionnaire was completed with the help of relatives and carers. This sought full unit postcodes for all places of residence, education, and employment from birth to onset of disease as well as general life profile data. Data were obtained from 130 patients with MND (77 males and 53 females, male/female ratio 1.45). The analyses described 
Table 1 The UK Postcode: basic principles and concepts

\begin{tabular}{lll}
\hline Postcode level (example) & Hierarchical description & Average population covered \\
\hline PR $^{\star} \star \star \star$ & Area & 461250 \\
PR2 $\star \star \star$ & District & 20580 \\
PR2 9 & Sector & 6200 \\
PR2 9HT & Full unit postcode & 38 \\
\hline
\end{tabular}

in this paper are restricted to residences within the postcode areas BB (Blackburn), FY (Blackpool), LA (Lancaster), and PR (Preston). These areas were estimated to cover a population of 1473153 in the 1991 census and cover the essential catchment area of the Department of Neurology in Preston. These designations are, however, only a general guide to the zone covered by each postcode area. LA postcodes cover the area well to the north of Lancaster, stretching to Barrow-in-Furness in the north west; similarly, BB postcodes relate to Burnley as well as the urban area of Blackburn. Although patients inevitably spent some time living outside these four postcode areas at various times in their lives the methodology described here is aimed at studying the distribution of place of residence of patients with MND in the BB, FY, LA, and PR postcode areas. It is not concerned with detailed migration history.

Because of the hierarchical nature of the UK postcode (table 1) we may form counts of cases within postcode areas (such as LA, BB, and so on), postcode districts (LA1, LA2, and so on), or postcode sectors (LA1 3, LA2 4, etc). This paper describes analyses at postcode district and sector level. The numbers of places of residence of patients with MND in these specific areal units are compared with expected theoretically derived values which assume a uniform distribution across the BB, FY, LA, and PR postcode areas. The theoretically derived values were calculated from the 1991 UK Census using population data for each areal unit. Expected values were thus calculated for the number of MND patient residences (equation) for each of the postcode districts and sectors in the BB, FY, LA, and PR postcode areas. This enables an expected value for each areal unit to be obtained which takes account of its specific population.

$$
\begin{aligned}
& \text { Expected number } \\
& \text { of places of residence }
\end{aligned}=\frac{\mathrm{n}_{\text {resid }} \times \mathrm{p}_{\text {areal unit }}}{\mathrm{P}}
$$

where

$\mathrm{n}_{\text {resid }}=$ total number of MND patient residences in BB, FY, LA, and PR postcode areas (482)

$\mathrm{p}_{\text {areal unit }}=$ population of areal unit (postcode district or sector)

$\mathrm{P}=$ total population of $\mathrm{BB}, \mathrm{FY}, \mathrm{LA}$, and $\mathrm{PR}$ postcode areas (1 473 153).

\section{Results}

The 130 patients with MND identified in this study imply an incidence of $1.76 / 100000$ population/year. This is in accordance with previous literature estimates and suggests a satisfactory level of case ascertainment. The 130 patients lived 5462 years in 482 residences in the 52 postcode districts and 216 postcode sectors in the BB, FY, LA, and PR postcode areas. The postcode districts and sectors with extreme residuals are shown in table 2 .

No MND residences were found in nine out of 52 postcode districts (17\%) and 59 out of 216 postcode sectors (27\%). Although these data suggest that the background incidence of MND may be relatively low they do not take account of the wide variation in population between the areal units. This impression is, however, supported when the numbers of MND places of residence were compared with population expected values. When this was done (table 3) it was found that up to three times as many areal units have one or less MND places of residence than expected at district and sector levels.

This thesis can be further developed by examining the frequency distribution of the residuals (MND-expected population value). This is shown for the district based analysis in fig 1 and for the sector based analysis in fig 2 . The median residual is a negative value for both the district and sector analysis $(-1.15$ for the district analysis, -0.35 for the sector analysis). This again suggests that more areal units (both at district and sector level) have a lower rather than an increased prevalence of MND places of residence. Statistically these results are unsurprising; they are consistent with the positively skewed distribution which would normally be expected of count data. The key statistical question is whether the pattern found could have arisen by chance rather than evidencing a systematic spatial variation in MND residence spells.

Poisson modelling was used to test for random variation. The explicit assumption of this analysis was that the expected number of MND residences would be proportional to population size. The Poisson model provided a poor fit for the data at postcode district level (goodness of fit $\left(\chi^{2}=217\right.$ with 51 degrees of freedom) indicating that patients with MND were more likely to have lived in some districts than others even after allowing for variation in postcode district population. However, before accepting this result as evidence of spatial clustering of risk factors, it is important to eliminate possible alternative explanations. There are two main possibilities.

(1) The pattern may be a spurious result due to some districts having a more rapid population turnover than others. We investigated this by including mean duration of residence as an explanatory variable in the Poisson model. The effect was not significant (goodness of fit $\left(\chi^{2}=0.02\right.$ with 1 degree of freedom (df)) and the model continued to show substantial variation in MND rates between districts (goodness of fit $\left(\chi^{2}=216.3\right.$ with $\left.50 \mathrm{df}\right)$.

(2) The significance of the goodness of fit test may have been inflated by individual residential moves within the same district. To investigate this effect, we reanalysed the data treating multiple residence spells in a district as a single count. The goodness of fit statistic was now only significant at the $10 \%$ level $\left(\left(\chi^{2}=65.6\right.\right.$ with $51 \mathrm{df})$. However, this is a conservative test 
Table 2 Summary of raw data, residuals, and ratios from representative postcode districts and sectors

\begin{tabular}{|c|c|c|c|c|c|c|c|}
\hline & \multirow[b]{2}{*}{ Population } & \multicolumn{2}{|c|}{ Number of residences } & \multirow[b]{2}{*}{ Residual } & \multirow{2}{*}{$\begin{array}{l}\text { Ratio (observed/expected) } \\
\text { with } 95 \% \text { CIs }\end{array}$} & \multicolumn{2}{|c|}{$\begin{array}{l}\text { Duration of residence } \\
\text { (person residence years) }\end{array}$} \\
\hline & & Observed & Expected & & & Observed & Expected \\
\hline \multicolumn{8}{|l|}{ District: } \\
\hline BB3 & 31161 & 34 & 10.20 & 23.80 & $3.33(2.31-4.66)$ & 294.80 & 115.54 \\
\hline BB5 & 57460 & 36 & 18.80 & 17.20 & $1.91(1.34-2.65)$ & 429.76 & 213.06 \\
\hline FY7 & 26202 & 20 & 8.57 & 11.43 & $2.33(1.43-3.60)$ & 162.24 & 97.16 \\
\hline PR7 & 45269 & 26 & 14.81 & 11.19 & $1.76(1.15-2.57)$ & 260.24 & 167.85 \\
\hline BB1 & 59118 & 30 & 19.40 & 10.60 & $1.55(1.04-2.21)$ & 411.46 & 219.21 \\
\hline BB6 & 20086 & 17 & 6.57 & 10.43 & $2.59(1.51-4.14)$ & 146.59 & 74.48 \\
\hline FY1 & 36046 & 21 & 11.79 & 9.21 & $1.78(1.10-2.72)$ & 194.55 & 133.66 \\
\hline BB7 & 19521 & 14 & 6.39 & 7.61 & $2.19(1.20-3.68)$ & 178.57 & 72.38 \\
\hline LA13 & 18890 & 0 & 6.18 & -6.18 & $0(0.00-0.60)$ & 0 & 70.04 \\
\hline LA9 & 25567 & 2 & 8.37 & -6.37 & $0.24(0.03-0.86)$ & 13.88 & 94.80 \\
\hline LA14 & 41567 & 6 & 13.59 & -7.59 & $0.44(0.16-0.96)$ & 61.56 & 154.05 \\
\hline PR4 & 51345 & 7 & 16.80 & -9.80 & $0.42(0.17-0.86)$ & 77.86 & 190.38 \\
\hline PR9 & 39351 & 1 & 12.88 & -11.88 & $0.08(0.00-0.43)$ & 3.16 & 145.91 \\
\hline PR8 & 50836 & 3 & 16.63 & -13.63 & $0.18(0.04-0.53)$ & 13.01 & 188.50 \\
\hline \multicolumn{8}{|l|}{ Sector: } \\
\hline BB5 5 & 9136 & 15 & 2.99 & 12.01 & $5.02(2.81-8.27)$ & 121.74 & 33.88 \\
\hline BB6 9 & 7496 & 13 & 2.45 & 10.55 & $5.31(2.83-9.07)$ & 125.40 & 27.79 \\
\hline BB3 0 & 6846 & 11 & 2.24 & 8.76 & $4.91(2.45-8.79)$ & 91.6 & 25.38 \\
\hline PR1 5 & 4397 & 8 & 1.44 & 6.56 & $5.56(2.40-10.90)$ & 42.90 & 16.30 \\
\hline BB7 1 & 6246 & 7 & 2.04 & 4.96 & $3.43(1.38-7.07)$ & 101.61 & 23.16 \\
\hline BB3 1 & 6819 & 7 & 2.23 & 4.77 & $3.14(1.26-6.47)$ & 90.69 & 25.28 \\
\hline FY1 4 & 7221 & 7 & 2.36 & 4.64 & $2.97(1.19-6.11)$ & 28.99 & 26.77 \\
\hline BB3 2 & 7677 & 7 & 2.51 & 4.49 & $2.79(1.12-5.75)$ & 73.24 & 28.47 \\
\hline BB5 6 & 8135 & 7 & 2.66 & 4.34 & $2.63(1.06-5.42)$ & 83.68 & 30.16 \\
\hline PR7 4 & 385 & 4 & 0.13 & 3.87 & $30.8(8.38-78.8)$ & 59.93 & 1.43 \\
\hline PR2 2 & 98 & 2 & 0.03 & 1.97 & $66.7(8.07-241)$ & 1.12 & 0.36 \\
\hline FY1 3 & 3839 & 5 & 1.26 & 3.74 & $3.97(1.29-9.29)$ & 56.62 & 14.23 \\
\hline BB1 7 & 3892 & 5 & 1.27 & 3.73 & $3.94(1.27-9.16)$ & 33.77 & 14.43 \\
\hline PR5 7 & 3140 & 4 & 1.03 & 2.97 & $3.88(1.06-9.97)$ & 44.90 & 11.64 \\
\hline PR1 7 & 12598 & 0 & 4.12 & -4.12 & $0(0.00-0.90)$ & 0 & 46.71 \\
\hline PR1 9 & 17967 & 1 & 5.88 & -4.88 & $0.17(0.00-0.95)$ & 22.01 & 66.62 \\
\hline PR9 7 & 17030 & 0 & 5.57 & -5.57 & $0(0.00-0.66)$ & 0 & 63.15 \\
\hline PR2 3 & 28242 & 1 & 9.24 & -8.24 & $0.11(0.00-0.60)$ & 8.38 & 104.72 \\
\hline
\end{tabular}

Key: Highest, Lowest.

Table 3 Summary of basic analysis of data at postcode district and sector levels

\begin{tabular}{|c|c|c|c|c|}
\hline & Observed (95\% CIs) & Expected $(95 \%$ CIs $)$ & Odds ratio & $95 \%$ CIs of odds ratio \\
\hline \multicolumn{5}{|l|}{ District level ( $\mathrm{n}=52$ districts): } \\
\hline One or less $\mathrm{MND}$ residence & $14(8-21)$ & $6(2-12)$ & 2.33 & $0.97-5.60$ \\
\hline More than one MND residence & $38(31-44)$ & $46(40-50)$ & & \\
\hline Negative residual & $33(25-40)$ & & & \\
\hline Positive residual & $19(12-27)$ & & & \\
\hline \multicolumn{5}{|l|}{ Sector level ( $\mathrm{n}=216$ sectors): } \\
\hline One or less MND residence & $107(93-121)$ & $33(23-43)$ & 3.24 & $2.30-4.56$ \\
\hline More than one MND residence & $109(95-123)$ & $183(173-193)$ & & \\
\hline Negative residual & $124(110-138)$ & & & \\
\hline Positive residual & $92(78-106)$ & & & \\
\hline
\end{tabular}

because people who moved between districts are counted more than once and must tend to dilute any spatial clustering due to risk factors ${ }^{\star}$; we interpret the result as providing further evidence of a genuine spatial clustering.

The whole set of statistical analyses was repeated at postcode sector level. The results are less reliable with these data because of the larger number of areal units with a zero or very low value. Nevertheless, the poor fit of the Poisson model tended to support the trends found in the postcode district data (goodness of fit $\left(\chi^{2}=732.6\right.$ with $215 \mathrm{df}$ allowing for sector population size, $\left(\chi^{2}=709.7\right.$ with 214 df allowing for sector population size and mean length of residence and $\left(\chi^{2}=530.8\right.$ with $205 \mathrm{df}$ when multiple residence spells within sectors are counted singly).

We therefore conclude that there is prima facie evidence of systematic spatial patterns in the residence histories of patients with MND.

${ }^{\star}$ We are grateful to an anonymous referee for pointing this out.
In particular, this evidence indicates that the counts are more dispersed than random, implying more low counts and more high counts than would be expected by chance if MND rates were spatially homogeneous in the population. As the distribution of residence counts is positively skewed these results support the conclusion from the descriptive analysis. The average number of residences is disproportionately influenced by a relatively small number of areas with high residence counts.

The postcode district BB5 showed an apparent excess of MND residences. Within this district the sector BB5 5 contains $15(42 \%)$ of the 36 BB5 MND places of residence. Assuming a uniform distribution of MND places of residence across the four postcode areas BB, FY, $\mathrm{LA}$, and PR a figure of 3.0 (BB5 5) out of 18.8 (BB5) - that is, $16 \%$ - would be expected. The postcode district $\mathrm{BB} 6$ shows a similar excess. Within the BB6 district 13 (35\%) of the 17 BB6 MND places of residence were within the BB6 9 sector. Assuming a uniform distribution 


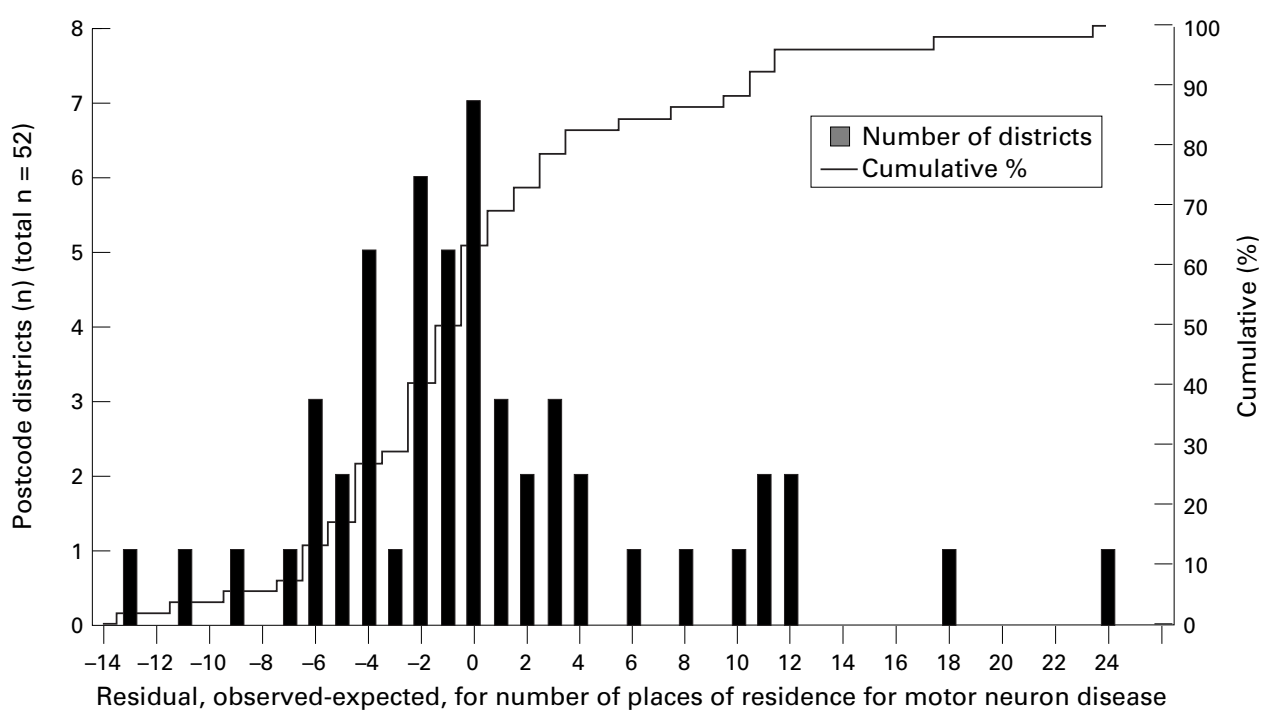

Figure 1 Frequency distribution of residuals from analysis by postcode district.

of MND places of residences a figure of 2.5 out of $6.6(19 \%)$ would be expected. The areal units showing relatively low MND prevalence include the LA14, PR4, LA9, and LA13 districts along with the PR1 9 and PR1 7 sectors.

If environmental factors operating in zones such as BB5, BB6, and others do influence susceptibility to MND the results so far described do not imply an "all or nothing effect". The possibility that duration of residence or period of residence (window of exposure effect) might also be important in the context of determining susceptibility to MND has not yet been discussed. An attempt to address this question is shown in fig 3 for four postcode districts. This figure shows duration and periods of residence in BB3, BB5, BB6, and FY7. Examination of these figures does not suggest that residence at any particular time in history was a common factor for the persons living in these districts who went on to develop MND although seven of the eight patients lived in BB5 did so between the mid-1950s and
1990. Eight of the nine living in BB3 resided in the district between the late 1970s and 1980s. It is also noted that some patients had several different residences within the same areal unit and that one patient had 10 places of residence within the BB5 5 sector.

\section{Discussion}

We have conducted an investigation of the geographical distribution of MND patient residences in Lancashire and south Cumbria. We have compared the geographical distribution of the residences of these patients with MND with population based reference data derived from the 1991 UK Census.

As would be expected on a priori grounds the results show that areas exist where more MND residences have occurred as well as other areas in which fewer MND residences have occurred than expected. We would thus provide statistical modelling evidence that this effect is more accentuated than would be expected by chance. Although much attention has been previously given to possible

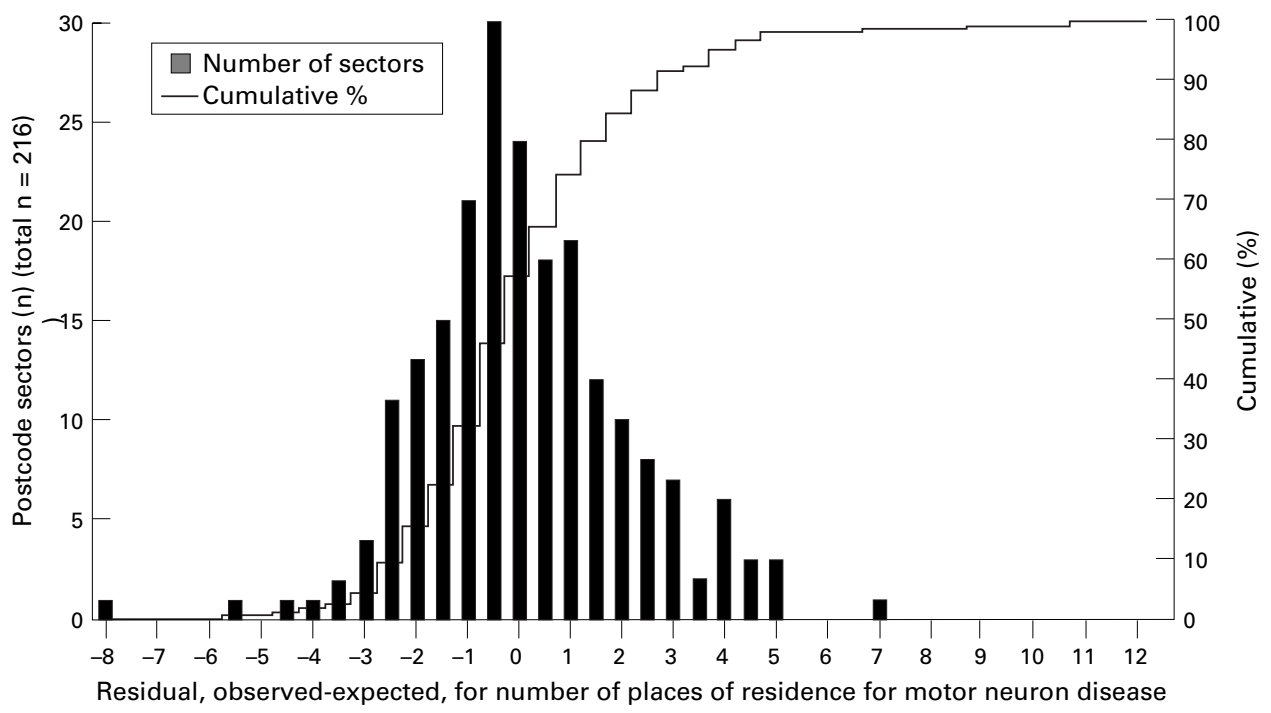

Figure 2 Frequency distribution of residuals from analysis by postcode sector. 
geographical concentrations of MND (clusters), little attention has been given to the possibility that areas might also exist with a relatively low risk of MND. The results presented here are probably the clearest indication to date that such areas might exist.

The findings, particularly at postcode sector level, suggest a relatively low background incidence of MND residences. With a positively skewed distribution of residence counts there is an excess of sectors showing a negative rather than a positive residual and the number of sectors with one or less places of MND residence is substantially greater than expected. This suggests that high incidence foci of MND increase the overall incidence of the disease from a relatively low background figure towards the rather higher values generally reported in the literature.

A particular aggregation of MND residences was found in the BB5 postcode district. Further analysis indicates that this aggregation was focused within the BB5 5 postcode sector within that district. This area comprises a small town with outlying rural areas. Further aggregations of $M N D$ residences were found in the BB3 (BB3 0, BB3 1, and BB3 2 sectors), BB6 (BB6 9 sector), and FY7 postcode districts. The postcode district BB3 covers an industrial town dependent on wallpaper and paint manufacturing to the south of Blackburn with surrounding elevated moorland. The sectors BB3 0, BB3 1, and BB3 2 cover contiguous areas comprising the town centre and areas to the west. The BB6 9 sector covers a small country town to the north of Blackburn with extensive surrounding rural hinterland to the north. The FY7 district encompasses a town in the north Fylde adjacent to an estuary with

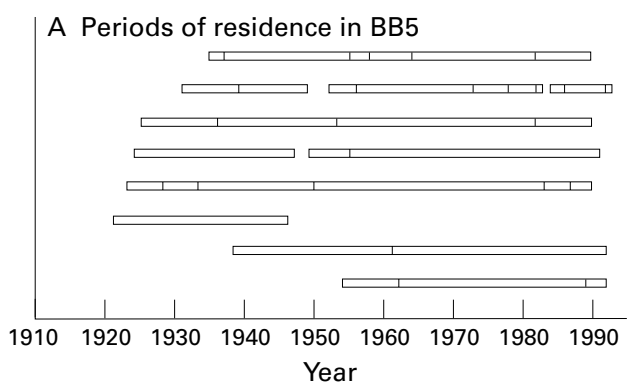

Data from 8 patients who had resided in 36 BB5 residences before disease onset

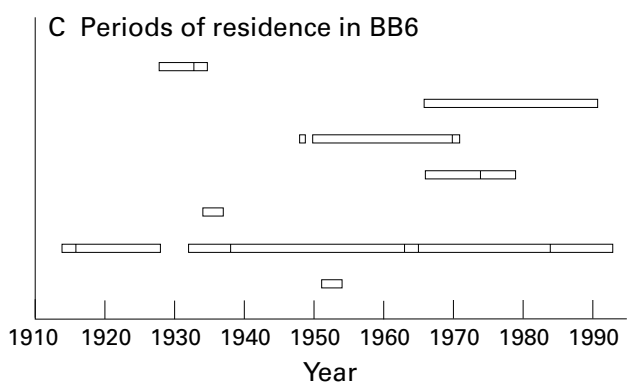

Data from 7 patients who had resided in 17 BB6 residences before disease onset extensive chemical installations. A sector within the FY7 district (FY7 8) emerged as a potential focus of an increased risk of MND in an earlier study. ${ }^{2}$

It is possible that these findings reflect different occupational, or other exposures, and are not necessarily related to place of residence. We also have complete data for places of education and employment similar in scope to the residential data reported here. We intend to report these results separately at a later date.

Earlier work using point process methods to detect clustering among MND patients in Lancashire and south Cumbria, over a 14 year period $(1976-89)^{2}$ failed to find convincing evidence that places of residence of patients with MND tended to cluster relative to controls. However, rather an ad hoc set of controls was used in this work, simply sampling at random from the Central Postcode Directory. ${ }^{4}$ Moreover, this previous work was based on address at the time of diagnosis, thus ignoring the previous residential histories of patients. Given the fact that peak age at diagnosis is between 60 and 70 years of age and that the mean duration of stay for patients with MND at each place of residence was 11.3 years patients are likely to have changed address several times before the disease becomes clinically manifest. This work thus extends earlier findings ${ }^{6}$ that were based on an analysis of patient data at electoral ward level. This previous work showed several wards with significantly high numbers of cases, but no information on duration of residence was available.

It should also be remembered that clustering might be determined by "duration of exposure" rather than the "all or nothing effect" implied by merely examining number of places

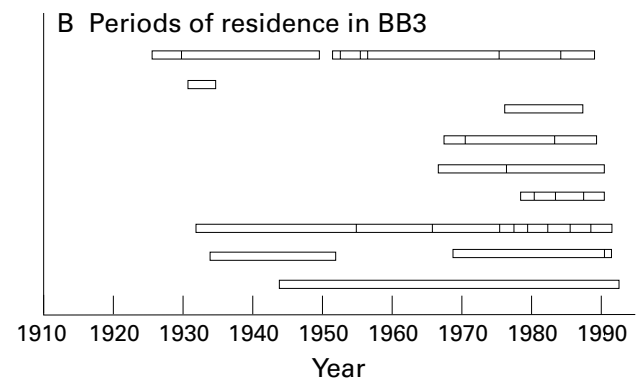

Data from 9 patients who had resided in 34 BB3 residences before disease onset

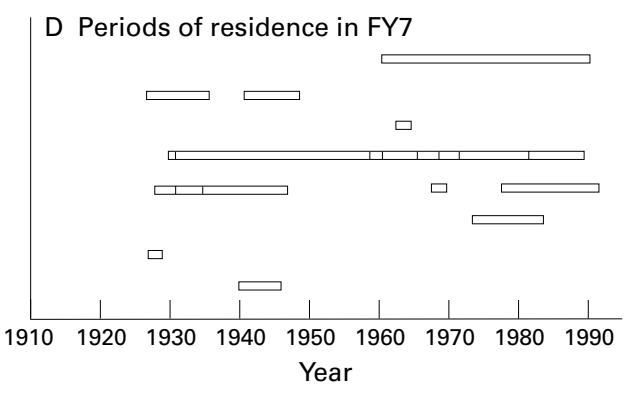

Data from 8 patients who had resided in $20 \mathrm{FY} 7$ residences before disease onset

Figure 3 Residences for motor neuron disease in BB5, BB3, BB6, and FY7. (A) periods of residence in BB5; (B) periods of residence in BB3; (C) periods of residence in BB6; (D) periods of residence in FY7. 
of residence as has been done here. It is thus also important to consider the total time of residence at each location as shown in fig 3. We have full details of dates and duration for each place of residence, education, and employment for the 130 patients with MND and a more detailed analysis of these issues will follow in further work. However the mean stay for patients with MND at each place of residence in BB3, BB5, BB6 and FY7 was comparable with an overall mean stay of 11.3 years.

Other attempts to detect clustering among patients with MND have been limited. At a very fine geographical scale, both Hochberg et $a l^{7}$ and Melmed and Krieger ${ }^{8}$ found "clusters" of three patients who had lived in close proximity (either in the same apartment building or same block of houses). The absence of data on suitable controls renders these essentially anecdotal reports difficult to evaluate. After detection of a possible cluster of six cases in Two Rivers, Wisconsin, Sienko et al sought to determine factors that might increase the relative risk; physical trauma and the consumption of fish caught from Lake Michigan were two significant factors. In Wisconsin as a whole, evidence points to a clear excess of cases in the north east of the state ${ }^{10}$ reasons for which are unclear; however, no age standardisation was performed and those counties with high rates are thinly populated rural areas with very small numbers of cases. ${ }^{11}$

On a broader scale, studies in the United States ${ }^{1}$ have found an excess of counties with above average mortality from MND in areas west of the Mississippi, and other work ${ }^{12}$ has shown a very clear gradient in terms of birthplace of cases, states in the north west United States (for example, Washington, Montana, and Idaho) showing increased rates compared with the country as a whole. In south west Ontario ${ }^{13}$ some spatial variation in incidence was found, but this was not significant. In Finland, ${ }^{14}$ data from a 10 year period (1963-72) indicated significantly raised incidence in two regions in the south east of the country. In Scotland ${ }^{15}$ significantly higher rates were found in districts to the north and east of the country, though a more detailed study in the Edinburgh (Lothian) region $^{16}$ failed to identify any postcode districts with significantly increased disease risk. In an examination of mortality rates by county, Buckley et $a l^{17}$ found some evidence of higher rates in the south, and a study in northern England at the scale of health authorities ${ }^{18}$ detected high standardised incidence rates in some districts, although the failure to attach confidence intervals and the small numbers of cases throughout the study area renders the result of doubtful interest.

\section{Conclusions}

The results presented here follow the screening of an extensive dataset of patients with MND for evidence of spatial clustering of place of residence. Evidence has emerged suggesting that geographical areas exist where more MND patient residences have occurred than would have been expected. This reinforces some previous work in this area, much of which has tended to be qualitative rather than quantitative. Areas have also been found where fewer MND patient residences have occurred than would have been expected. It is suggested that the general background incidence of MND is low in the context of generally quoted incidence figures, but that this overall incidence is skewed upwards by the relatively high incidence areas to achieve the generally quoted figures for the incidence of MND. The occurrence of this apparently uneven distribution of MND residences is reinforced by the results of Poisson modelling. There is a clear need to further examine occupational and environmental factors in the lives of the patients with MND and reference subjects to obtain a better understanding of the relevance of these findings. We intend to develop the analysis of this dataset in this way in the hope that it might enable us to gain further insights into factors which determine the incidence of MND and thus improve our understanding of the pathogenesis of one of the most tantalising diseases known to medicine.

This work was funded by the Motor Neurone Disease Association, UK, particularly the Manchester Branch. We thank them for their generous support without which this work would not have been possible. We are also indebted to the Royal Mail for their generous donation of a set of UK Postcode Directories for their generous donat
to assist in this work.

1 Bharucha NE, Schoenberg BS, Raven RH, et al. Geographic distribution of motor neuron disease and correlation with possible etiologic factors. Neurology 1983;33:911-15.

2 Mitchell JD, Gibson HN, Gatrell AC. Amyotrophic lateral sclerosis in Lancashire and South Cumbria, 1976-86. Arch Neurol 1990;47:875-80.

3 Brooks BR. El Escorial World Federation of Neurology criteria for the diagnosis of amyotrophic lateral sclerosis. $f$ Neurol Sci 1994;124(suppl):96-107.

4 Gatrell AC, Dunn CE, Boyle PJ. The relative utility of the central postcode directory and pinpoint address code in applications of geographical information systems. Environment and Planning A 1991;23:1447-58.

5 Raper J, Rhind DW, Shepherd J. Postcodes: the new geography. Longman, Harlow, 1992.

6 Gatrell AC, Mitchell JD, Gibson HN, et al. Tests for spatial clustering in epidemiology: with special reference to motor neurone disease. In: F Clifford Rose, ed. Advances in ALS/ $M N D$. London: Smith-Gordon, 1991.

7 Hochberg FH, Bryan JA, Whelan MA. Clustering of amyotrophic lateral sclerosis. Lancet 1974;i:34.

8 Melmed C, Krieger C. A cluster of amyotrophic lateral sclerosis. Arch Neurol 1982;39:595-6.

9 Sienko DG, Davis JP, Taylor JA, et al. Amyotrophic lateral sclerosis: a case-control study following detection of a cluster in a small Wisconsin community. Arch Neurol 1990;47: $38-41$.

10 Taylor JA, Davis JP. Evidence for clustering of amyotrophic lateral sclerosis in Wisconsin. F Clin Epidem 1989;42:56975.

11 Martyn CN. Epidemiology. In Williams A, ed. Motor neurone disease. London: Chapman and Hall, 1994:393-426.

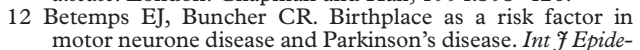
miol 1993;22:898-904.

13 Hudson AJ, Davenport A, Hader WJ. The incidence of amyotrophic lateral sclerosis in southwestern Ontario. Neurology 1986;36:1524-8.

14 Jokelainen $M$. The epidemiology of amyotrophic lateral sclerosis in Finland. $\mathcal{F}$ Neurol Sci 1976;29:55-63.

15 Holloway SM, Emery AEH. The epidemiology of motor neuron disease in Scotland. Muscle Nerve 1982;5:131-3.

16 Holloway SM, Mitchell JD. Motor neurone disease in the Lothian region of Scotland, 1961-81. F Epidemiol Community Health 1986;40:344-50.

17 Buckley J, Warlow C, Smith P, et al. Motor neuron disease in England and Wales, 1959-79. F Neurol Neurosurg Psychiatry 1983;46:197-205.

18 Qizilbash N, Bates D. Incidence of motor neurone disease in the northern region. $f$ Epidemiol Community Health 1987;41:18-20. 